Editorial

\title{
Food Structure Design: Innovation in food structure-properties relationships
}

The food industry is constantly challenged to meet consumer demands for new food products that are safe, convenient, affordable, pleasurable and healthy. An understanding of fundamental structure and function relationships of food components is a key to the design of new foods: ingredient formulation and production processes have a major role in commercial food development but their impact on food structure is poorly characterized.

The goal of the Congress on Food Structure Design is to strengthen at academic and industrial levels the scientific understanding of product design and engineering, and to stimulate and accelerate the development of innovative, complex and highly structured products and suitable production processes.

The main topic of the 1st edition of the Congress has been therefore the design of health-supporting food functions with development of food structure/health benefits relationships in complex real multiphase structured foods with optimal sensory quality.

This conference was meant to gather an interdisciplinary team of scientists from different research areas (food engineering, biophysics, applied soft matter, food technology, applied human nutrition, creative design) in the frame of an integrated process $\&$ product design approach for creating innovative multiphase structured foods.

Sharing knowledge and technologies for healthy foods design among academic institutions and private enterprises for the purpose of further development has also been addressed.

The Congress was developed on three directions of "Food Structure Design":

I. Engineering of structures for tailored delivery of healthrelated functionalities

II. Process and product engineering for food properties generation/preservation/delivery

III. Sharing knowledge and technologies between academia and industry for healthy foods design

With the following sub-topics:

I. Engineering of structures for tailored delivery of healthrelated functionalities

Elucidating structure-property functions and designing model multiphase food systems a. Biophysics/Biochemistry-driven strategies for structure design in model multiphase foods

b. Engineering self-assembly nano- and microstructures in food systems

c. Designing complex (bio)polymer and colloidal systems

d. Designing of novel and/or tunable structures for engineered nano- and microstructures for stability and controlled release of bioactives

e. Development of advanced and appropriate analytical and theoretical tools to elucidate the effects of structure on the health and nutritional attributes of the final food product

II. Process and product engineering for food properties generation/preservation/delivery

Assessing the role of processing in stabilizing of complex multiphase real foods or new food ingredients and in reformulating the nutrient contents of foods to enhance their nutritional profile

a. Understanding and controlling the behavior of functional ingredients/ingredient design with targeted health functionality

b. Redesign of traditional processes to meet new consumer needs

c. Biophysical and gastro-intestinal engineering aspects of nutrient absorption and physiological function

d. Process innovations in encapsulation technology, drying, structuring technologies, non-thermal processing following a criterion for structure/process design

e. Structure-sensory properties relationship and consumer perception as a criterion for process design

f. Meeting consumer sensorial demands and nutritional needs through rational design of structures and processes

III. Sharing knowledge and technologies between academia and industry for healthy foods design

Bridging the innovative Food Structure Design approach with the criteria for the market driven food research

a. New concepts and processes for creating healthy foods

b. Structure design for gastronomy applications

c. Enterprise-based innovation system in Food Structure Design

d. Case studies on structure/process development with relevance to different food manufacturing processes 
e. Transferring skills, knowledge, technologies to private companies: role of Technology Transfer Mediators and of Transfer Agencies

Overall participation has resulted in 10 plenary and keynote lectures, 23 oral presentations and over 140 posters, in a total of 37 countries represented.

This Special Issue gathers selected contributions from the 1 st Congress on Food Structure Design, covering most of the topics described above.

Cristina L.M. Silva CBQF - Centro de Biotecnologia e Química Fina - Laboratório Associado, Escola Superior de Biotecnologia,
Universidade Católica Portuguesa/Porto, Rua Arquiteto Lobão Vital, Apartado 2511, 4202-401 Porto, Portugal E-mail address: clsilva@porto.ucp.pt

António A. Vicente Centre for Biological Engineering, Universidade do Minho, Campus de Gualtar, 4710-057 Braga, Portugal

Laura Piazza

Department of Food Environmental and Nutritional Sciences, Università degli Studi di Milano, via Celoria, 2 - 20133 Milano, Italy 